\title{
La enseñanza de las lenguas a través de la transdisciplinariedad como un reto de la postmodernidad
}

\author{
Language teaching by transdisciplinarity as a postmodern challenge \\ Claudia Gil-de la Piedra ${ }^{a}$
}

\begin{abstract}
:
This paper aims to problematize the strategies which are used to teach foreign languages in Mexican research and higher education centers, in the frame of globalization, and in order to emphasize the importance of languages in the new social context. Likewise, we intend to highlight the reciprocal importance of academic and pedagogic research in order to develop language teaching. At the same time, the knowledge and proficiency of a foreign language facilitates academic research in Mexico. This study proposes transdisciplinarity as a means of reconstructing and contextualizing a subject matter according to specific students' needs to promote academic and cultural exchange in Mexican higher education. By this means, research will be inserted into academic debates in the global worldwide.
\end{abstract}

\section{Keywords:}

Contextualizing, higher education, globalization, epistemic thought, post-modernity, transdisciplinarity

\section{Resumen:}

El objetivo general de este trabajo es problematizar las estrategias de enseñanza de lenguas extranjeras en las instituciones de educación superior en México, en el marco de la globalización, así como enfatizar la importancia de las lenguas en los nuevos contextos sociales. Del mismo modo, se pretende resaltar la importancia recíproca de la investigación académica y pedagógica para el avance de la enseñanza lingüística, así como la relevancia que tiene el conocimiento y dominio de una lengua extranjera para desarrollar la investigación académica en México. Este estudio propone la transdisciplinariedad como un medio de reconstruir un objeto de estudio y contextualizarlo en función de necesidades específicas de los estudiantes para favorecer el intercambio académico y cultural en la educación superior mexicana y, de este modo, insertar la investigación mexicana en los debates académicos del mundo global.

\section{Palabras Clave:}

Contextualización, educación superior, globalización, pensamiento epistémico, posmodernidad, transdisciplinariedad

\section{LA POSMODERNIDAD COMO MARCO DE LA PROBLEMÁTICA}

El aprendizaje lingüístico y la investigación académica tienen sus bases en los conceptos desarrollados en las teorías difundidas mediante procesos de enseñanza-aprendizaje a nivel superior. Este trabajo persigue la hipótesis de que es posible implementar una nueva forma de cuestionar los conceptos adquiridos a través de una segunda lengua para enmarcarlos en nuestra actualidad y en nuestro país, si se tienen en cuenta los cambios sociales, económicos y estructurales del pensamiento global, en un contexto específico. De este modo, es posible asimilarlos en nuestro contexto real y crear un aprendizaje real y significativo. En el caso del proceso de enseñanza-aprendizaje de lenguas extranjeras en México, es necesario delimitar las problemáticas existentes en relación con el bajo rendimiento en las instituciones de educación superior y las consecuencias que éste tiene en relación con el desarrollo de la investigación académica. Asimismo, es indispensable contextualizar el proceso de aprendizaje lingüístico para poder analizarlo a detalle y proponer un cambio sustancial que tienda a reformular objetivos y metodologías dentro de las aulas.

Para empezar a abordar el tema, es necesario tener en cuenta que la posmodernidad es un concepto que aún no ha sido bien definido; con frecuencia, se le confunde con un periodo histórico que da continuidad a la modernidad. Sin embargo, según el sociólogo Zygmunt Bauman (2007), este término hace referencia a un complejo sistema de ideas y a las prácticas y costumbres que nos definen actualmente; es decir, a la caída de las Instituciones sociales establecidas, a la pérdida de valores y de verdades absolutas, así como a la descentralización del conocimiento. En consecuencia, estos cambios han tenido un profundo impacto en los procesos de enseñanza-aprendizaje. 
Para poder enmarcarlos, es pertinente retomar el análisis de Bauman, On education (2012), donde se plantea la necesidad de una verdadera revolución cultural del aprendizaje dentro de la globalización.

El análisis de Bauman define nuestra época a partir de la heterogeneidad y descentralización del pensamiento mediante una constante interacción, gracias al planteamiento de la globalización. Bauman (2007) explica que "en la conciencia posmoderna no hay salida cierta de la incertidumbre" (p. 412). Por esta razón, la posmodernidad implica un cambio de perspectiva, así como de subjetividad. Asimismo, representa una nueva mirada hacia los otros que desvía la atención de aquello que se considera perfecto y concede importancia a lo que es diferente y periférico. Esto tiene un fuerte impacto en el ámbito educativo de nuestra época debido a la reformulación de objetivos y a la necesidad de delinear metodologías alternativas que nos permitan dialogar con los estudiantes para transmitir conocimiento que pueda ser funcional en su cotidianidad y que pueda adaptarse a sus necesidades específicas.

Es pertinente cuestionar el uso que se da a una lengua extranjera, llamada así por ser ajena en nuestra tradición cultural y en nuestro territorio. No obstante, si su utilidad reside únicamente al ser hablada fuera de nuestro marco, habría que poner en duda si la lengua extranjera no es en realidad la localidad y nosotros los verdaderos extranjeros, ajenos al contexto de ésta y, a no ser que viajemos al territorio de esa lengua, donde somos nosotros los extranjeros, la lengua no tendrá mucho valor. El objetivo del aprendizaje debe encontrar una verdadera utilidad, que sirva igualmente como motivación, para que la importancia de una lengua extranjera sea visible en cualquier territorio. De la misma manera, es importante determinar cuáles son los factores contextuales que influyen en el aprendizaje y cuál es el impacto ideológico que se produce en el mundo debido a la globalización. Esto nos lleva a redefinir la importancia del conocimiento teórico, de cómo desarrollarlo y cómo formular cuestionamientos que ayuden a la formación de futuros profesionistas, sin apelar únicamente a la repetición mecanizada de la teoría occidental, sino, de igual modo, construyendo argumentos propios.

¿Cuáles son los principales retos que plantea la globalización en materia de aprendizaje lingüístico? Podemos comenzar argumentando que la globalización, siendo un modo de integración, está estrechamente ligada al pensamiento posmoderno pues éste, en su afán de reafirmar la diferencia y la heterogeneidad, ha dado cabida a la diversidad y a la pluralidad cultural, a la emergencia de discursos subalternos, de distintas razas, géneros y creencias, lo cual permite establecer nuevos vínculos y nuevas formas de conocimiento. La posmodernidad formula una nueva ideología que deconstruye los esquemas precedentes y tradicionales. Antes, se podía definir la cultura como "un conjunto de problemas y temas, modos de percibir y de razonar, hábitos mentales y códigos retóricos comunes a una sociedad y que hacen posible la comunicación cultural dentro de ella" (Altamirano, 1980, p. 16). Estos comportamientos estaban ligados a la determinación colectiva, y reflejados bajo la forma de "bienes culturales". Esta cultura englobada en la modernidad permanecía sólida y defendía la certeza de las verdades absolutas. Contrariamente a esta idea, la posmodernidad implica una existencia vacía de certeza que Bauman ha llamado "modernidad líquida". Este concepto evidencia la caída de la totalidad de la ciencia, de la estabilidad humana y de la solidez de las relaciones culturales e interpersonales. "La cultura líquida moderna ya no siente que se trata de una cultura de aprendizaje y acumulación como las civilizaciones registradas en los datos de los historiadores y etnógrafos. En cambio, parece una cultura del desapego, de la descontinuidad y del olvido" (Bauman, 2012, p. 46).

Frente a esta situación, es necesario modificar los programas educativos, sobre todo aquellos que se centran en la cultura y la lengua, a partir de la pluralidad cultural, ideológica y lingüística que ha surgido a raíz de la globalización y de este nuevo esquema de pensamiento. Sin embargo, es preciso tomar en cuenta que la propuesta de Bauman se enunció en Europa, al igual que mucha de la investigación académica, y que no es analizada en América Latina hasta que es traducida, años después de su publicación. Por lo tanto, cabe hacer notar la imperativa necesidad de una propuesta teórica que cuestione el entorno a partir de la percepción mexicana y del contexto académico en el que se enuncia. Por esta razón, la enseñanza de lenguas extranjeras adquiere un carácter fundamental en la formación profesional de los estudiantes de cualquier nacionalidad, ya que, gracias a la comprensión de otras lenguas, el académico es capaz de insertarse en los debates críticos y en el intercambio educativo global.

\section{LA TRANSDISCIPLINAREIDAD Y EL PROCESO DE APRENDIZAJE}

Desde una perspectiva global, la transdisciplinariedad proporciona una diversidad de enfoques sobre un mismo objeto de estudio. Como señala Basarab Nicolescu (1996), "la transdisciplinariedad comprende, como el prefijo trans lo indica, lo que está entre las disciplinas, a través de las diferentes disciplinas y más allá de toda disciplina. Su finalidad es la comprensión del mundo presente, y uno de sus imperativos es la unidad de conocimiento" (p.37). Nicolescu apela a distintos planos de realidad, lo cual da sentido a todo aquello que se encuentra entre y a través de las disciplinas. Esta diversidad de planos de realidad se hace más visible en el contexto posmoderno, donde se perciben diferentes concepciones del mundo que se encuentran en intercomunicación constante. Esto quiere decir que la transdisciplinariedad nos permite apelar a distintos puntos de vista y, en consecuencia, nos lleva a ampliar el horizonte de análisis; es un ejercicio dialéctico donde el objeto es sometido a un proceso de resignificación constante.

La enseñanza de lenguas permite abordar diferentes temas, colaborar con otras disciplinas y refleja las diferencias culturales, así como el modo de expresarlas. La lengua funciona, la mayor parte del tiempo, como un mediador, como un puente 
a través del cual se transmite el conocimiento. En consecuencia, además de ser un objeto de estudio en sí mismo, es también un constructor de ideas. A partir del lenguaje se define una cosmovisión y se define una tradición. La lengua evoca la cultura y el lugar de enunciación desde donde se aporta un sistema de pensamiento. Por lo tanto, ahondar en una lengua es también profundizar en lo que hay a través y más allá de diferentes disciplinas que constituyen nuestro objeto de estudio profesional (biología, mecánica, química, etc.) y cómo son percibidas en otros territorios. Por consiguiente, la enseñanza-aprendizaje de una lengua extranjera no está limitada a su conocimiento disciplinar, ni es un accesorio de la vida profesional. Ésta debe ser una herramienta fundamental de la educación superior en México y el mundo vinculada con cualquier disciplina.

Sin embargo, debido al cambio social constante y a sus repercusiones en el pensamiento colectivo, existe un desfase entre la manera de percibir la realidad y la manera de asirla, comprenderla y reexplicarla. El investigador chileno Hugo Zemelman (2002) explica los motivos de este desfase: "el uso de conceptos sin una clara significación provoca la necesidad constante de resignificación en los procesos de investigación y metodología" (p. 1); esto quiere decir que la sociedad se transforma más rápidamente que las teorías que se formulan para comprenderla. Los profesores habían decidido establecer una distancia histórica, que consideraban favorable, para hacer un análisis objetivo. Zemelman pone en duda si esta distancia es, en efecto, pertinente. Podríamos seguir su ejemplo, ya que nos enfrentamos a una realidad que se nos presenta incomprensible y aterradora debido al aumento de la aceleración del tiempo histórico. En ocasiones, los conceptos que utilizamos han sido ya rebasados o han cambiado su significado. Por ejemplo, en ciertas disciplinas como la biología, la literatura y la lingüística existen nuevos métodos de análisis que evidencian formas innovadoras de aprehender la realidad desde la perspectiva de los estudios profesionales. La rápida evolución de la tecnología, la nueva maquinaria y la nueva distribución del trabajo han hecho surgir otro tipo de relaciones humanas, otras palabras; es decir, neologismos y, al mismo tiempo, otros contextos.

En lo que concierne a las lenguas extranjeras, el intercambio constante entre las tradiciones culturales hace más fáciles las transformaciones ideológicas. Éstas tienden a un cosmopolitismo que podríamos llamar, de acuerdo con Bauman (2012), “mixofilia”. Ésta consiste en el interés por las mezclas raciales y culturales. Ésta misma constituye un ejemplo de neologismo que ha surgido en razón de una modificación de la realidad global. La asimilación y adopción de nuevos términos que hacen referencia a las costumbres y tradiciones extranjeras, expresiones idiomáticas o simples neologismos, forman parte de una lengua y de su estructura. La lengua, de la misma forma que un ser vivo, puede evolucionar e hibridarse con otros rasgos lingüísticos proporcionados por diversos factores como la migración, la guerra o los medios de comunicación masiva. Zemelman acentúa que, si no se toman en cuenta los riesgos de este desfase, terminaremos por "pensar ficticiamente en realidades inventadas, enmarcadas en un pensamiento inventado que no corresponde a nuestra vida cotidiana" (2002, p. 2).

Es preciso preguntarse de qué manera es posible otorgar un nuevo significado al concepto de enseñanza-aprendizaje lingüístico dentro de la educación superior, teniendo en cuenta el cambio ideológico y social que nos rodea. Hay que tomar en cuenta que, de la misma manera que los conceptos migran de un país a otro o de una clase social a otra, es necesario que éstos sean asimilados según el marco del grupo social que los recibe y que la resignificación de los mismos se genere apelando a las diferencias culturales y a la visión de mundo para saber cómo será incluido el concepto en la cultura meta. De esta manera, es posible el diálogo de dos o más disciplinas, no sólo en sus similitudes, sino también en sus diferencias y lo que éstas engloban.

El pensamiento posmoderno define la diferencia como herramienta enriquecedora. Éste es el marco donde se desarrolla la construcción de un nuevo conocimiento teórico basado en el diálogo y donde las lenguas extranjeras se posicionan como disciplina, como medio de comprender, de construir, de enunciar y de replicar y, a su vez, como objeto de estudio. Es pertinente plantearse si, en realidad, los profesores nos asumimos en realidad como ciudadanos del mundo, así como lo proponen los nuevos métodos de educación global, o si, a decir verdad, seguimos reproduciendo los estereotipos vigentes que marcan cada país. Esto no se trata de la pérdida de la identidad nacional, sino de cuestionar la eficacia del lenguaje dentro de la escena global.

Es importante pensar en crear una red de conceptos globales que puedan adquirir un nuevo significado en función de la realidad actual para formular teorías e hipótesis que expliquen nuestro mundo para entenderlo mejor. Un ejemplo de esta tentativa es el aprendizaje significativo enunciado por Grant Wiggins (2005), quien propone crear unidades de enseñanza definidas por un tema que unifique una serie de conceptos significativos, centrados en un área de interacción común para diversas materias. De esta manera, podemos partir de una pregunta que haga surgir el interés de los alumnos, pero, al mismo tiempo, que los incite a relacionar los conceptos usados en clase con su vida real y sus problemas cotidianos. De igual modo, se establece una relación con otras materias escolares que pueden abordar los mismos conceptos desde otras perspectivas. Un ejemplo de esto es el método de enseñanza del Bachillerato Internacional. El objetivo más importante del BI en relación con la enseñanza de Lengua B es "permitir a los alumnos desarrollar competencias de multiculturalidad y las actitudes necesarias para dar muestra de competencias interculturales para poder comunicarse eficazmente en los contextos mundiales del siglo XXI" (Guía Lengua B, 2013, p. 2).

Otro ejemplo de aprendizaje global es la multialfabetización, descrita por Frida Díaz Barriga, quien afirmó que "la multialfabetización consiste en desarrollar las competencias cognitiva y comunicativa de los educandos en el más amplio sentido, hacerlos educandos y educables, e incluso, yendo más 
allá, posibilitar el desarrollo social, cultural y político de la sociedad o comunidad cultural de la comunidad en la que se encuentran" (Díaz, 1990, p. 273).

Sin embargo, este proceso no es del todo fácil de llevar a la práctica. Este aprendizaje requiere del desarrollo del pensamiento crítico en los alumnos. Éste último otorga la posibilidad de expandir el horizonte cultural y reducir los prejuicios surgidos a partir de las diferencias. Es necesario comprender que el estudio de una lengua extranjera no se trata de comunicar encontrando una equivalencia con el horizonte cultural de la lengua materna, sino de abrir la perspectiva hacia los otros.

\section{LOS NIVELES DE APRENDIZAJE Y LA INFRA EDUCACIÓN}

Según Gregory Bateson (1998), existen tres niveles de aprendizaje. El primero consiste en la transferencia de información para la memorización. Este nivel parece ser el más fácil y, en la mayoría de los casos de educación básica, es el más empleado por los enseñantes, debido a la tradición de la modernidad de conservar y acumular: ya sean bienes materiales o bienes culturales, dinero o memoria. No obstante, como he explicado, la modernidad ha empezado a perder sus valores y su validez a gran velocidad, frente a la incertidumbre planteada por la posmodernidad. De esta manera, la memoria ha perdido igualmente su valor y el estudiante se encuentra varado entre la pérdida de su memoria, la manipulación de los medios de comunicación y la incomprensión de los cambios que suceden a su alrededor. En este nivel de aprendizaje, el estudiante escucha la verdad absoluta enunciada por el profesor y la memoriza. Existe una distancia vertical entre el enseñante y el alumno que impide al último cuestionar la información adquirida. Precisamente, en los últimos años, los enseñantes se han pronunciado contra esta metodología. Pero, ¿cómo concebir la metamorfosis de la educación básica adquirida bajo este modelo educativo hacia la apertura de pensamiento que se espera de los universitarios?

El segundo nivel propuesto por Bateson es el deuteroaprendizaje. Éste implica la necesidad de dominar un cuadro cognitivo específico; la información percibida ha sido ya asimilada e incorporada a la estructura mental del estudiante; es el aprendizaje del aprendizaje. Sin embargo, no se llega a profundizar en el objeto de estudio desde todas sus perspectivas. En consecuencia, existe el riesgo del aplicacionismo, que consiste en aplicar una teoría específica, como si se tratara de una receta de cocina, sobre un objeto de estudio. Esto quiere decir que se quiere encontrar una perfecta similitud entre la teoría y la realidad, lo cual limita la capacidad crítica de los alumnos e, igualmente, impide reformular las ideas en función de necesidades concretas. Es posible que mucha gente piense que éste no es su caso, aunque caer en este error es más fácil de lo que se piensa. La admiración por el genio, por la teoría y por todo lo que ha sido definido como cultura dentro de un canon social afecta nuestras decisiones y limita nuestro horizonte de percepción y, en consecuencia, de aprendizaje.

El tercer nivel mencionado por Bateson implica actitudes que permiten el cuestionamiento del cuadro cognitivo planteado. Éste representa "una profunda reorganización del carácter" (1998, p. 331). Es preciso, en este momento, deconstruir y reconstruir el objeto de estudio en función de nuestro contexto y de la construcción social que nos ha definido y formado. Aun cuando este nivel cognitivo representa un gran avance para el pensamiento humano, llegar a este punto involucra todo un proceso psicológico y pedagógico que permite ampliar la visión del objeto. Este tipo de aprendizaje es indispensable para el desarrollo del pensamiento humano, para la formación profesional y para el desarrollo de la investigación académica.

Bauman hizo notar, a principios del siglo XXI, que "estamos en tiempos de pánico, pues hay un incremento de gente infra educada; ésta es el resultado de la baja de recursos universitarios y de la disminución del número de titulados de buena calidad" (2012, p. 59). Bauman evidencia la elevada cifra de titulados en condición de desempleo y el papel de las universidades, públicas o privadas, que aumentan cada vez más el número de titulados mecanizados que se quedan dentro de los dos primeros niveles cognitivos descritos por Bateson (1998). Éstos obtienen su título universitario pero no producen un conocimiento significativo ni una aportación real. Es posible entender esto mediante la perspectiva de Grant Wiggins quien afirma que "el significado no se puede enseñar; es necesario que éste sea diseñado por el estudiante via artful design y por un acompañamiento eficaz por parte del profesor" (2005, p. 104). Si bien es cierto que Bauman habla de las universidades inglesas, la situación no es menos grave en América Latina.

Para hacer frente a este problema, Hugo Zemelman argumentó a favor de la creación de un pensamiento americano encaminado a entender la realidad local dentro de su contexto. Esto implica construir un punto de vista sin los prejuicios y preconcepciones del pensamiento eurocentrista. En México, en el caso de la enseñanza de lenguas, hay que preguntarse por qué la mayoría de estudiantes que empieza a tomar un curso de lengua extranjera no lo termina. ¿Por qué si se estudia el inglés desde la escuela primaria, los mexicanos no hablan inglés? Según Juan Pardinas, director del Instituto Mexicano para la Competitividad (IMCO), sólo el 5\% de la población habla inglés en México (Becerril, 2015). Otras fuentes como el EF English Proficiency Index indican que México tiene un nivel bajo en relación con su nivel de aptitud. La clasificación mexicana se sitúa en 49.76 (EF EPI, 2018), por debajo de países de América del Sur, como Chile y Argentina, que no tienen un contacto directo con Estados Unidos. Es innecesario decir que el caso del francés es aún peor. El inglés forma parte de los programas educativos de las escuelas primarias públicas desde hace casi diez años. Sin embargo, los resultados no han sido positivos. La asimilación del mundo extranjero a partir de libros de texto ilustrados en función de la realidad europea o estadounidense no conlleva la identificación del sujeto con su objeto de estudio, que es, en este caso, la 
lengua. Se apela a la lengua francesa evocando la belleza de los Campos Elíseos, la moda y la Torre Eiffel; pero cabe preguntarse si éstos son argumentos suficientemente poderosos para demostrar a los alumnos la importancia de la lengua francesa si, quizá, no van a viajar a Francia por el momento. Así que ¿cuál es la importancia de aprender inglés o francés en México? Una lengua extranjera no es un lujo ni una herramienta de viaje. Es preciso resaltar la importancia de las lenguas extranjeras aun cuando el estudiante no sale del país.

Siendo un estudiante universitario, un individuo tiene acceso a un cierto número de artículos virtuales, libros y bases de datos. Pero, la mayoría de estas bases están en inglés o en francés y, con frecuencia, no se encuentran traducciones, sobre todo si las publicaciones son muy recientes. Por este motivo, los estudiantes no conocen la existencia de esta información, lo cual nos lleva a la falta de información especializada en las universidades mexicanas. Es necesario comprender que el aprendizaje lingüístico no es un requisito para ir de vacaciones, sino un requisito para la información y comunicación del mundo global. Se estudia en libros de crítica literaria, de sociología, etc., traducidos en España o en Argentina, que llegan a México cinco años después de su publicación original. Esta situación enfatiza el problema del desfase señalado por Zemelman: los alumnos empiezan a estudiar a partir de una realidad teórica ya rebasada. Otro problema dentro del aprendizaje de lenguas extranjeras es el uso de conceptos que han adquirido otro significado. Zemelman también afirma que se pueden utilizar los mismos conceptos desde diversos puntos de vista, pues "existe una capacidad diferente de formular los atributos de los fenómenos y dar significaciones diferentes" (2002, p. 8) Para ilustrar esto, tomaré como ejemplo el término avant-garde. Éste puede ser explicado desde diversas disciplinas: artística, de la moda, militar, educativa, etc.; y en cada una podemos imaginar un ícono distinto. La representación del lenguaje depende de la construcción social de la persona que lo interpreta. Siempre estamos expuestos a la interpretación de la realidad y el lenguaje es en sí mismo una traducción de lo que observamos. Por esta razón, el pensamiento crítico, que permite distinguir los fenómenos que dañan o que construyen la sociedad, resulta indispensable. La única forma de hacer esta distinción es a través del lenguaje y se expresa mediante la lengua. De este modo, se justifica la enorme importancia de la enseñanza de las lenguas extranjeras para la comprensión de otros entornos y del propio. Así como hemos planteado los problemas fundamentales para el aprendizaje universitario en México, enmarcado en la posmodernidad; podremos proponer algunas alternativas formuladas como sugerencias para agilizar el proceso de aprendizaje.

\section{UNA NUEVA PROPUESTA INTERCULTURAL A PARTIR DE LA CONSTRUCCIÓN Y DELIMITACIÓN DE CONTEXTOS}

Si se afirma que la ideología posmoderna ha deconstruido los esquemas tradicionales, habría entonces que deconstruir la idea de jerarquía imperante en los salones de clase universitarios. Esto consiste en reducir la distancia entre el enseñante y el estudiante. No se busca romper con los valores de respeto y disciplina, sino de establecer un diálogo entre ambos. Normalmente, este diálogo se establece con el objeto de estudio; es decir, objeto-sujeto. Es importante considerar que no se trata de una relación lineal, sino de un triángulo de comunicación. Si bien el alumno absorbe el conocimiento del enseñante y del objeto que estudia, es a partir de la retroalimentación que el enseñante permanece en constante aprendizaje, incorporando la experiencia y la percepción del estudiante. Estas dos modifican siempre la significación dada al objeto de estudio. El enseñante debe estar atento a estas transformaciones del pensamiento y contextualizar la clase en función del alumno y del objeto de estudio. Dicha propuesta implica que no se puede usar el mismo plan de clase para dos grupos diferentes, aún si se trata del mismo tema, ya que cada estudiante tiene una perspectiva y una percepción distinta y, por lo tanto, sus necesidades y su proceso de aprendizaje serán diferentes.

Mientras tanto, el objeto de estudio; es decir, la lengua, está en constante cambio y reformulación, comunica y dialoga de acuerdo con el contexto de aprendizaje. De esta manera, todos los actores son activos en este triángulo de comunicación. Ya no es una relación lineal, vertical o centralizada, sino circular. Para ejemplificar mejor esto, digamos que la lengua estudiada se transforma y se resignifica; ya no es simplemente un objeto de estudio, sino una productora de conocimiento y transformadora de modos de pensar.

Una vez explicado esto, hay que cuestionar la utilidad del aprendizaje. La realidad es que el alumno no retendrá lo aprendido si no es útil para él. Por ejemplo, si se aprenden los nombres de los animales del bosque tropical o todo tipo de frutas y verduras, es poco probable que el estudiante llegue a utilizar este léxico en una conversación fluida o en la búsqueda de información útil para sus estudios. Actualmente, existen métodos especializados para ciertos tipos de estudiantes universitarios, como de negocios o turismo, pero quizá hace falta un método que tenga en cuenta el contexto del estudiante mexicano.

Esta propuesta se trata de contextualizar el aprendizaje lingüístico y de enmarcarlo en la realidad mexicana. Es necesario entender el contexto como un concepto siempre cambiante al ritmo de la sociedad que enmarca, y no como un límite rígido de tiempo y espacio. Silvia Pappe (2002) definió el contexto como "un reflejo de nuestra actual percepción acerca de los horizontes que abarca un objeto de estudio en un momento dado" (p. 30). Entender este enunciado es fundamental para la investigación, la enseñanza y el aprendizaje, ya que siempre es necesario contextualizar y enmarcar un objeto de estudio. El hecho de señalar el espacio y la época en los que viven los estudiantes de una lengua no es suficiente; tampoco lo es determinar la estructura específica de una lengua. De lo que se trata, más bien, 
es de plantear todos los campos de referencia mediante los cuales se puede analizar y la manera en que interactúan. Pappe resalta que contextualizar quiere decir: organizar, acotar, definir, explicar, significar, concretizar, dar forma, interpretar, pero sobretodo, comprender (2002, p. 34).

Puesto que el pensamiento debe adaptarse creativamente a los cambios históricos, debe estudiarse el mayor número de perspectivas posibles. Para contextualizar un objeto de estudio, nos es precisa una variedad de enfoques. Por ejemplo, es preciso definir por qué es necesario estudiar una lengua, cuándo, cómo y dónde se usará este conocimiento, y cuál será el impacto del uso de la lengua en la vida cotidiana y profesional del individuo que la aprende. Asimismo, debe delimitarse la diferencia entre el modo de usar la lengua y la importancia que ésta tiene en un contexto local; es decir, su uso como lengua extranjera, o en un contexto donde el hablante es el extranjero, ya que el uso de la lengua varía desde dónde y cómo se enuncia.

La lengua no es rígida ni lineal, es un sistema, una estructura que existe en diferentes realidades al mismo tiempo y que desempeña diversos papeles. Un ejemplo en el caso del francés, es la diversidad de fonéticas, expresiones idiomáticas y contenidos culturales englobados en los países francófonos. Existe, en cada país, una construcción cultural diferente y una visión de mundo propia de la comunidad. Este marco de diferencias construye el horizonte de la lengua de estudio y la inscribe dentro de la globalización. La lengua francesa se reafirma como un objeto de estudio vivo y cambiante, manifestándose de distintas maneras a lo largo del mundo, globalmente. De este modo, la rigidez lingüística da paso a la heterogeneidad del discurso francófono y a las voces emergentes de países fuera de la centralización francesa.

\section{CONCLUSIONES}

Debe tenerse en cuenta que las lenguas deben estudiarse de acuerdo con las épocas y los procesos sociales e históricos variables relacionados con éstas. Resulta fundamental establecer la pertinencia del aprendizaje de las lenguas extranjeras, así como de su investigación y su difusión en relación con el esquema de pensamiento posmoderno, ya que es en función de éste que se define su uso y su importancia. Siempre es necesario proporcionar a los estudiantes objetivos específicos que los motiven.

Finalmente, retomaré la sugerencia de Hugo Zemelman, quien propone distinguir entre el pensamiento crítico -el cuál es totalmente necesario- del pensamiento epistémico. Éste último rebasa al primero debido a que consiste en encontrar el origen del conocimiento. Si bien el pensamiento crítico es indispensable para cuestionar la realidad, "el pensamiento epistémico nos permitirá profundizar, abrirse a nuevas perspectivas" (2002, p. 4). Como Zemelman explica, el pensamiento epistémico nos permite confrontar nuestra realidad y plantear problemas. De acuerdo con Zemelman, el problema más grave es la construcción de hipótesis a partir de ideas predicativas; es decir, puramente descriptivas. Las ideas son los atributos de un objeto de estudio. En oposición a esto, el pensamiento epistémico plantea al objeto de estudio sin un contenido de atributos. Éste es el pensamiento vacío, aquel que nos lleva a formular cuestionamientos y servir como guía de aprendizaje.

El pensamiento epistémico señalado por Zemelman hace referencia a la manera de aprehender la realidad que se percibe y de buscar nuestro propio referente de interpretación, original y auténtico. Si la lengua forma parte de nuestra personalidad y es esencial en la conformación de nuestra humanidad, es indispensable que se desarrolle a partir de nuestra propia percepción del mundo. La lengua es una herramienta para la construcción del pensamiento epistémico, pero si se trata de una lengua extranjera, ésta también puede desempeñar un papel como constructora de conocimiento lingüístico, cultural y como puente que une dos visiones de mundo.

Para concluir, quisiera enfatizar la importancia de estos cuestionamientos para desarrollar el aprendizaje. A pesar de que he centrado mi argumento en la enseñanza de las lenguas, debido a que es el tema que me concierne, el pensamiento epistémico puede ayudarnos a construir todo tipo de análisis en cualquier disciplina. En México, aún se está desarrollando un sistema eficaz de enseñanza-aprendizaje centrado en las lenguas extranjeras. Para lograrlo, es necesario seguir trabajando teniendo en cuenta la contextualización de los receptores, de los emisores y del objeto de estudio; es decir del hablante, del aprendiente y de la lengua misma. Esto se refiere a la construcción del contexto, la cual se hace posible y más eficaz si se apela a otras disciplinas que puedan dotar de significado al proceso de aprendizaje. Es necesario enfatizar la necesidad de las lenguas extranjeras para acceder a la educación superior y, del mismo modo, es menester vigilar la evolución del proceso de desarrollo linguiístico a través de los niveles educativos precedentes.

Puesto que el ser humano está constituido por diferentes aspectos, también su pensamiento está conformado por distintas facetas que se unifican para formar una visión de mundo. De esta manera, la transdisciplinariedad nos ayuda a proponer diferentes respuestas para una misma pregunta, tomando en cuenta las diferencias que nos rodean. La intención de este trabajo es señalar algunas problemáticas en el aprendizaje, pero también plantear preguntas, algunas de la cuales quedan todavía sin respuesta. Sin embargo, lo que propongo es apelar a la creación de una teoría del aprendizaje que se centre en nuestras verdaderas necesidades cognitivas como receptores de una segunda lengua, dentro del contexto mexicano. Es necesario empezar a citar a los críticos e investigadores mexicanos en nuestras universidades, pero primero, es preciso construirlos. Cabe también enfatizar la necesidad de una concepción de nuestra cultura desde nuestra propia perspectiva que nos permita hacer frente a la globalización de una manera eficaz sin perder nuestra identidad. Esto puede ser posible gracias a la relación de varias disciplinas que representan distintas miradas hacia nuestros estudios y que, al mismo tiempo, los enriquecen. La 
enseñanza transdisciplinaria desvela otras percepciones sobre los mismos conceptos y hace posible la construcción de una realidad individual original y auténtica. Al mismo tiempo, es un medio de diálogo. Si bien ayuda a comprender otras realidades, es un modo de hacerse visible para otros, de exportar y construir conocimiento.

\section{REFERENCIAS}

Altamirano, Carlos y Sarló Beatriz. (1980). Conceptos de sociología literaria. Buenos aires: Centro editor de América Latina.

Basarab Nicolescu. (1996). La Transdisciplinariedad: Manifiesto Traducción: Norma Nuñez Dentin. Ediciones Du Rocher. Recuperado de: http://ecosad.org/phocadownloadpap/otrospublicaciones/nicolescumanifiesto.pdf

Bateson, Gregory. (1998). Pasos hacia una ecología de la mente. Buenos aires: Lohlé-Lumen.

Bateson, Gregory. (1965). Comunicación, matriz social de la psiquiatría. Buenos aires: Paidós.

Bauman, Zygmunt. (2007). "Modernidad, ambivalencia y fluidez social" en: Las contradicciones culturales de la modernidad. Barcelona: Antrophos- México: UAM-A.

Bauman, Zygmunt. (2012). Sobre la educación en un mundo líquido. Barcelona: GEDISA.

Becerril, Isabel. (2015). "En México sólo un 5\% de la población habla inglés: IMCO”. Recuperado de: https://www.elfinanciero.com.mx/economia/en-mexico-solo-de-lapoblacion-habla-ingles-imco.html

Díaz Barriga, Frida. (1990). Metodología de diseño curricular para la educación superior. México: Trillas.

EF English Proficiency Index. (2018). Recuperado de: https://www.ef.com.mx/epi/

El programa de los años intermedios: Guía de Lengua B. (2012). Cardiff, Wales: Organización del Bachillerato Internacional.

Erickson, Lynn. (2014). Transitioning to concept- based Curriculum and instruction: how to bring content and process together. U.S.A.: Corwin.

Gil de la Piedra, Claudia. (2014). La nueva estructuración del programa de FLE dentro del Bachillerato Internacional (IB). México: UNAM.

McTigh, Jay y Grant, Wiggins. (2005). Understanding by design. Virginia: ASCD.

Nicolescu, Basarab. (1996) La transdisciplinariedad, Manifiesto. Hermosillo: Multidiversidad Mundo Real Edgar Morín A.C. Recuperado de: https://edgarmorinmultiversidad.org/index.php/descarga-libro-latransdiciplinariedad-en-manifiesto.html

Pappe, Silvia, en Ronzón, José y Jerónimo, Saúl. (2002). Reflexiones en torno a la historiografía contemporánea. México: Universidad Autónoma Metropolitana.

Zemelman, Hugo. (2002). Recuperado de: https://www.academia.edu/14214587/Pensar_Te\%C3\%B3rico_y_Pens ar_Epist\%C3\%A9mico_Los_retos_de_las_Ciencias_Sociales_Latinoa mericanas._Hugo_Zemelman. 